\title{
Improving Roamer Retention by Exposing Weak Locations in GSM Networks
}

\author{
Michal Ficek, Lukas Kencl \\ R\&D Centre for Mobile Applications \\ Czech Technical University in Prague \\ \{michal.ficek, lukas.kencl\}@rdc.cz
}

\begin{abstract}
Due to pricing policies set by network operators, roaming clients are among the most valuable clients of GSM cellular networks. Despite that each operator applies a lot of effort in network signal coverage in order to achieve the best conditions for roaming traffic in the network, there still remain hardly detectable locations where roaming clients are getting lost to rival networks. This paper presents a method for revealing roaming traffic weak locations, based on active tracking of roamers in a live GSM network. We define the problem scope using formal description of the active tracking process and propose a metric for evaluating network "weakness", together with intuitive visualization of weak locations. We shortly conclude with plans for ongoing research.
\end{abstract}

\section{Categories and Subject Descriptors}

C.2.3 [Computer-Communication Networks]: Network Operations-Network Monitoring

\section{General Terms}

Theory, Measurement

\section{Keywords}

GSM, cellular networks, active tracking, roaming

\section{INTRODUCTION}

Revenue from roaming in GSM networks, nowadays a widely used service, significantly contributes to network operators' profit. This fact forms a highly competitive environment in areas with one or more rival networks present, because unlike the domestic clients of the home network, a roamer can switch the network freely in places where the signal in the current network is lost and a rival network is thus considered better.

Besides weak signal coverage, operators' preference or managed-roaming settings, roaming retention can be negatively influenced by many unanticipated or simply overlooked causes. An essential precondition in finding out the reason for roamers' loss to rival networks is the knowledge of locations where it occurs. In a suspicious location (possibly covered by multiple cells of the GMS network) the operator can apply many methods for network improvement; in particular, the adjustment of the azimuth or tilt of the antennae, change of

Copyright is held by the author/owner(s).

CoNEXT Student Workshop'09, December 1, 2009, Rome, Italy. ACM 978-1-60558-751-6/09/12.

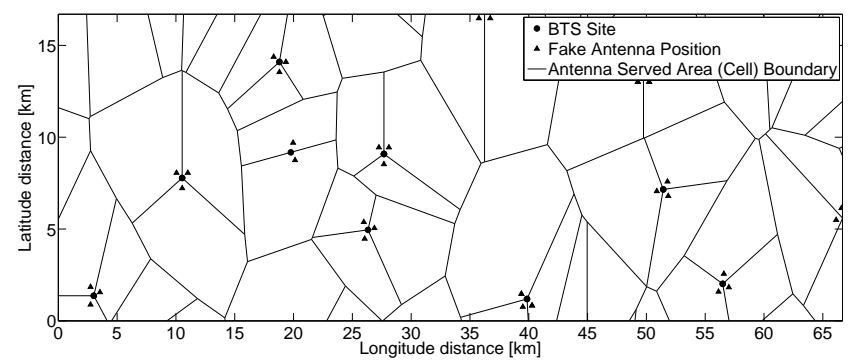

Figure 1: Antenna served area approximation based on Voronoi Tessellation [1]. For given cell $c$, corresponding Voronoi cell has been computed for nucleus with location of $l_{c}$ shifted by a little distance in a direction given by $a_{c}$. Although more sophisticated network approximations exist (e.g., [2]), we are limited by the two network parameters we have been given by the network operator.

signal power control or handover preference schema regulation can be applied. A last alternative may be building an extra cell tower, but being obviously costly, the lost revenue and return of investment must be carefully weighed.

Related work in roaming analysis consists mainly in commercial tools monitoring signaling interfaces in GSM networks and processing billing records $[6,7]$. In this paper we elaborate in detail an use-case of an active tracking platform, presented in [4].

\section{ACTIVE TRACKING FORMALIZATION}

Let $L \subset \mathbb{R}^{2}$ denote a set of sites (BTS locations) and let $C \subset \mathbb{N}$ be a set of all cell ID's in a studied network. For given cell identifier $c \in C$ we denote a cell an area served by a directional (rarely omni-directional) antenna located at site $l_{c} \in L$ with the direction azimuth $a_{c} \in\left[0^{\circ}, 360^{\circ}\right)^{1}$ (see Fig. 1).

Let $U \subset \mathbb{N}$ denote a set of users (roaming clients) subscribed to the studied network in the studied region. Let $S \subset \mathbb{N}$ denote a set of all possible user states: (1) subscribed to the studied network $\left(S_{s}\right),(2)$ subscribed to one of the rival networks in the studied region $\left(S_{r}\right)$ and $(3)$ other states $^{2}$. For simplicity, we consider only $S=\left(S_{s} \cup S_{r}\right)$ in this paper.

The output of active tracking of user $u$ is a timestamped history of state-cell relations $T^{u}=\left\{\left(t_{i}, s_{i}, c_{i}\right)\right\}_{i=1}^{n}$ described as follows:

\footnotetext{
${ }^{1}$ For omni directional antennas the azimuth parameter value can be arbitrary.

${ }^{2}$ States "offline", "abroad", "error" caused by GSM network unavailability, etc.
} 
- $t_{i}$ denotes a timestamp; consecutive timestamps' difference is not constant, i.e., $\left|t_{i+1}-t_{i}\right|=\delta+\Delta$, where $\delta$ denotes the fixed tracking interval and $\Delta$ denotes the variable network response time required for cell ID retrieval

- $s_{i} \in S$ denotes a state

- $c_{i}=\left\{\begin{array}{ll}c \in C & \text { if } s_{i}=S_{s} \\ & \text { if } s_{i} \in S_{r}\end{array}\right.$, i.e., the cell ID is available only for the studied network

Accordingly, we denote $T^{U}=\bigcup_{u \in U} T^{u}$ as active tracking of a set of users $U$. Then $\left(t_{i}^{u}, s_{i}^{u}, c_{i}^{u}\right)$ corresponds to $i$-th member of tracking $T^{u}$.

\section{PROBLEM STATEMENT}

The problem of revealing weak locations for roaming traffic is given as follows:

Given a set $L$ of site locations, a set $C$ of cell identifiers, and an active tracking $T^{U}$ of a set of users $U$, define an appraisal function $\mathbb{F}_{M}:\left(\mathbb{R}^{2} \times \mathcal{P}(C)\right) \rightarrow \mathbb{R}$ incorporating a cell-weakness metrics $M: C \rightarrow \mathbb{R}$.

Weak locations will then be determined as a set $W \subset \mathbb{R}^{2}$ satisfying $\forall x \in W: \mathbb{F}_{M}(x, A)>h$ for given threshold value $h$ and a set of cells $A \subseteq \mathcal{P}(C)$.

\subsection{Metrics}

Motivated by experiments on real data and considering two basic facts, (F1) users subscribed to a rival network will not generate any revenue for the operator of the studied network, and (F2) places visited by a non-trivial count of users are supposed to achieve earlier return of resources invested in the network enhancement, we propose a metrics $M$ characterizing "weakness" of a cell $c$ in the studied network in terms of roaming traffic as

$$
M(c)=\overbrace{\left|U_{c}\right|}^{\mathrm{F} 2} \cdot(\overbrace{\sum_{u \in U_{c}} \sum_{(i, j) \in I}\left|t_{j}^{u}-t_{i}^{u}\right|}^{\mathrm{F} 1})^{\alpha},
$$

where $U_{c}$ denotes a set of users which visited the cell $c$,

$$
U_{c}=\left\{u \in U|\exists i \in 1,2, \ldots,| T^{u} \mid: c_{i}^{u}=c\right\},
$$

a set $I$ contains indices of time intervals that the user $u$ spent in rival networks after visiting the cell $c$ until her return back to any cell in the studied network, i.e.,

$$
\begin{aligned}
I=\left\{(i, j)|i, j \in 1,2, \ldots,| T^{u} \mid, i<j ;\right. \\
\left.\quad c_{i}^{u}=c \wedge \forall k=i+1, i+2, \ldots, j: s_{k}^{u} \in S_{r} \wedge s_{j+1}^{u}=S_{s}\right\},
\end{aligned}
$$

and $\alpha \in \mathbb{R}$ is a parameter which adjusts the weighted influence of the time factor ${ }^{3}$ of the metrics.

Following real measurements in a live GSM network, some characteristics typical of roaming appeared. Time spent in a rival network is significantly higher on entry points to the network (especially near the studied country borders) because of operator preference stored on a roamers' SIM cards, but these values cannot be considered as outliers. For that reason, the time factor in equation 1 can be given less significance by applying e.g. the square root $(\alpha=0.5)$.

\footnotetext{
${ }^{3}$ Note that the time factor can be interpreted in the roamerday units, explained as one day spent in a rival network by one roamer; similarly to an industrial unit of production, man-day.
}

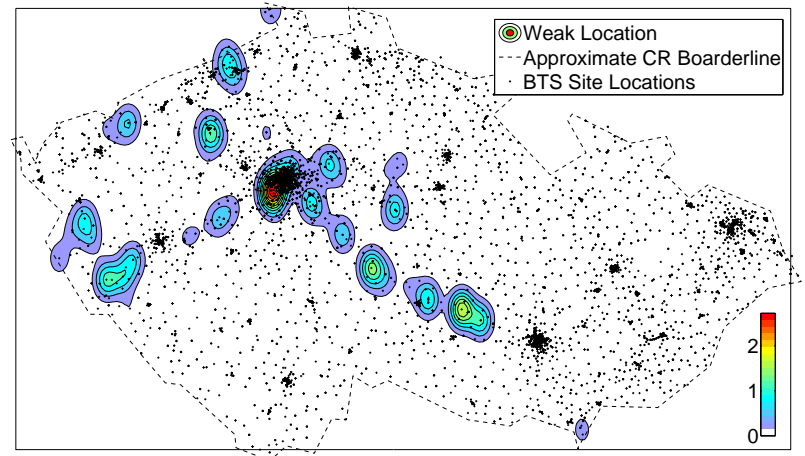

Figure 2: Visualization of suspicious weak locations within a GSM network of a large telecommunication operator in the Czech Republic, based on active tracking of 500 roaming users during 6 hours and evaluated using proposed metric $M$ with parameter $\alpha=0.5$. Colour scale corresponds to different threshold values. The data has been acquired during an experimental measurement with SS7Tracker platform [4].

\subsection{Weak Locations}

We choose the appraisal function to be a weighted kernel density estimator [5] with a Gaussian kernel and the proposed metrics $M$ as a re-weighting function. Intuitive visualization based on 2D-histogram, widely used in spatial data research (e.g.,[3]), has been employed in Fig. 2.

\section{CONCLUSION}

The method for exposing GSM cellular network weak locations with respect to roaming traffic, based on active tracking, presented in this paper as an early result of our research, represents a promising approach for detecting where important portion of operator's revenue is lost in the network. In immediate future we plan to employ a variable-kernel method [8] and kernel-shape change depending on a given cell shape and served-users' estimated location, followed by a scenario proposal for a statistically significant measurement.

\section{ACKNOWLEDGEMENT}

We wish to thank Vodafone Czech Republic a.s. for their generous support of the project.

\section{REFERENCES}

[1] F. Aurenhammer. Voronoi diagrams - a survey of a fundamental geometric data structure. ACM Comput. Surv., 23(3):345-405, 1991.

[2] F. Baccelli, B. Błaszczyszyn, and F. Tournois. Spatial averages of coverage characteristics in large cdma networks. Wirel. Netw., 8(6):569-586, 2002.

[3] M. Danese, M. Lazzari, and B. Murgante. Kernel density estimation methods for a geostatistical approach in seismic risk analysis: The case study of potenza hilltop town (southern italy). In ICCSA '08, Part I, pages 415-429, Berlin, Heidelberg, 2008. Springer-Verlag.

[4] K. Dufková et al. Active gsm cell-id tracking: "where did you disappear?". In MELT '08, pages 7-12, New York, NY, USA, 2008. ACM.

[5] F. J. G. Gisbert. Weighted samples, kernel density estimators and convergence. Empirical Economics, 28(2):335-351, 042003.

[6] Netcare International Ltd. Roaming tracker.

[7] Polystar OSIX AB. rdec (roam tracker).

[8] B. W. Silverman. Density Estimation for Statistics and Data Analysis. Chapman \& Hall/CRC, April 1986. 